\title{
Reply to comment on Lan et al.: S-osteotomy with lengthening and then nailing compared with traditional Ilizarov method
}

\author{
Xia Lan
}

Received: 26 November 2013 / Accepted: 28 November 2013 /Published online: 20 December 2013

(C) Springer-Verlag Berlin Heidelberg 2013

Dear Editor,

Obviously, the readers have paid close attention to our study

[1]. Thank you for all the questions and here are the answers:

1. The long $\mathrm{S}$ osteotomy with mini-incision is a highlyskilled technique. All operations in our centre were performed by senior surgeons. However, it can be performed more easily using a multiple-drill-hole technique. The details of the procedure has been described in the surgical technique part of the article. The anterior view of $\mathrm{S}$ osteotomy was demonstrated in Fig. 1a in the original article. Here is a photograph taken during the operation to show the mini-incision approach (Fig. 1).

2. Three to five weeks after the distraction phase ended, an intramedullary nail with full-length was inserted across the regenerated bone, and then the apparatus was removed. We chose the nail with appropriate diameter according to the size of the medullary canal, not a size 9 intramedullary nail for all patients. The skills to choose the appropriate nail and insert a nail with the external fixator in-situ have already been reported by Paley et al. [2] and Rozbruch et al. [3] and have been widely used for limb lengthening.

3. With the external fixator frame in-situ, it also requires considerable skill to insert a nail. This technique has already been reported by Paley et al. [2] and Rozbruch et al. [3]. To prevent deep infection and superficial pin-tract problems in our cases, all pins and wires were inserted without coming into contact with the intramedullary nail. This approach has also been reported by Paley et al. [2] and Rozbruch et al. [3].

Peifu Tang contributed equally to this paper.

\section{Lan $(\bowtie)$}

Department of Orthopaedics, Chinese People's Liberation Army

General Hospital, Beijing, China

e-mail: lanxjxh@126.com

X. Lan

The First Affiliation Hospital of Nanchang University, Nanchang city, Jiangxi Province, China

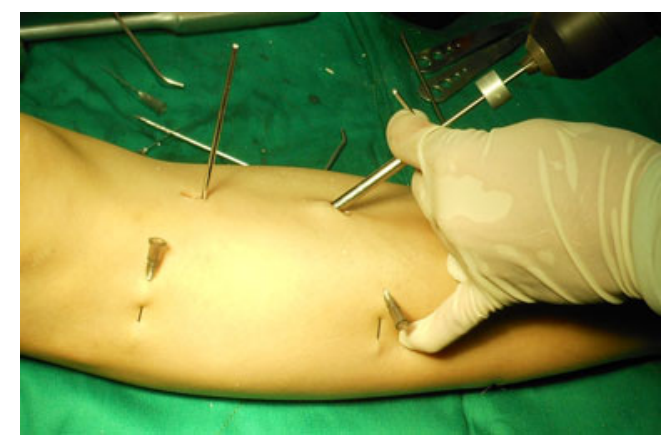

Fig. 1 Photograph taken during the operation to show the mini-incision approach

4. In our study, the indication for surgery in all patients was constitutional short stature, with no limb-length discrepancy before surgery. Furthermore, during the distraction phase, the rate of lengthening was adjusted according to the radiographs made every week, so the limb discrepancy could be completely avoided.

5. A detailed postoperative protocol has been described in the article. The postoperative protocols used in our study are routine protocols for limb lengthening. No other special postoperative protocol was followed to prevent ankle stiffness.

\section{References}

1. Lan X, Zhang L, Tang P, Xi H, Li G, Peng A, Han Y, Yuan B, Wenpeng $X$ (2013) S-osteotomy with lengthening and then nailing compared with traditional Ilizarov method. Int Orthop 37(10):1995-2000

2. Paley D, Herzenberg J, Paremain G, Bhave A (1997) Femoral lengthening over an intramedullary nail. A matched-case comparison with Ilizarov femoral lengthening. J Bone Joint Surg Am 79:1464-1480

3. Rozbruch SR, Kleinman D, Fragomen AT, Ilizarov S (2008) Limb lengthening and then insertion of an intramedullary nail: a casematched comparison. Clin Orthop Relat Res 466(12):2923-2932 